\title{
OPEN An animal model study on the gene expression profile of meniscal degeneration
}

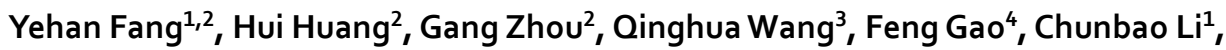
Yujie Liu ${ }^{1 \bowtie}$ \& Jianping Lin ${ }^{2}$

Meniscal degeneration is a very common condition in elderly individuals, but the underlying mechanisms of its occurrence are not completely clear. This study examines the molecular mechanisms of meniscal degeneration. The anterior cruciate ligament $(A C L)$ and lateral collateral ligament $(L C L)$ of the right rear limbs of seven Wuzhishan mini-pigs were resected (meniscal degeneration group), and the left rear legs were sham-operated (control group). After 6 months, samples were taken for gene chip analysis, including differentially expressed gene (DEG) analysis, gene ontology (GO) analysis, clustering analysis, and pathway analysis. The selected 12 DEGs were validated by real time reverse transcription-polymerase chain reaction (RT-PCR). The two groups showed specific and highly clustered DEGs. A total of 893 DEGs were found, in which 537 are upregulated, and 356 are downregulated. The GO analysis showed that the significantly affected biological processes include nitric oxide metabolic process, male sex differentiation, and mesenchymal morphogenesis, the significantly affected cellular components include the endoplasmic reticulum membrane, and the significantly affected molecular functions include transition metal ion binding and iron ion binding. The pathway analysis showed that the significantly affected pathways include type II diabetes mellitus, inflammatory mediator regulation of TRP channels, and AMPK signaling pathway. The results of RT-PCR indicate that the microarray data accurately reflects the gene expression patterns. These findings indicate that several molecular mechanisms are involved in the development of meniscal degeneration, thus improving our understanding of meniscal degeneration and provide molecular therapeutic targets in the future.
\end{abstract}

Meniscal degeneration is characterized by abnormal meniscus signals and extrusions at imaging, and it most often occurs in middle-aged and elderly patients with osteoarthritis. Meniscal degeneration has been regarded as one of the characteristics of osteoarthritis, but recent studies support that the normal meniscus has a significant protective effect on the articular cartilage ${ }^{1,2}$. Meniscal degeneration leads to loss of meniscal function, causing early-stage cartilage degeneration and subchondral bone loss, and leading to osteoarthritis and poor quality of $\operatorname{life}^{3,4}$. Therefore, delaying meniscal degeneration is considered important for preventing and treating osteoarthritis, but the specific molecular mechanisms for meniscal degeneration are not completely clear.

Due to ethics issues and limited donor menisci, especially normal control menisci, it is nearly impossible to conduct research on meniscal degeneration directly in humans, and appropriate animal models are required. Previous studies have often used small animals such as guinea pigs, rats, and rabbits for conducting research on meniscal degeneration ${ }^{5-9}$. Unfortunately, the animals themselves and their knee joints and menisci are small, and the results can hardly be translated to humans when considering the characteristics of the human knee joint weight-bearing and meniscal degeneration. The knee joint of mini-pigs has weight-bearing characteristics closer to that of humans.

Therefore, this study aims to examine the molecular mechanisms of meniscal degeneration. Based on the Pond-Nuki model of anterior cruciate ligament (ACL) resection ${ }^{10}$, the present study established models of meniscal degeneration using ACL and lateral collateral ligament (LCL) resection, and the gene chip analysis technology

\footnotetext{
${ }^{1}$ Medical School of Chinese PLA and Chinese PLA General Hospital, Beijing, China. ${ }^{2}$ Department of Orthopedic Surgery, Hainan General Hospital (Hainan Affiliated Hospital of Hainan Medical University), Haikou, Hainan, China. ${ }^{3}$ Department of Nursing, Hainan General Hospital (Hainan Affiliated Hospital of Hainan Medical University), Haikou, Hainan, China. ${ }^{4}$ Department of Sports Injury and Arthroscopy Surgery, National Institute of Sports Medicine, Beijing, China.『email: docman19@aliyun.com; linescu@163.com
} 
was used to study the changes in early meniscus degeneration gene expression profiles, providing evidence for studying the possible mechanism and treatment of meniscal degeneration.

\section{Materials and methods}

Pig model of meniscal degeneration. Seven Wuzhishan mini-pigs (Institute of Animal Science and Veterinary Medicine, Hainan Academy of Agricultural Sciences), with a mean age of $6.8 \pm 0.3$ (range: 6.1-7.4) months and a mean body weight (BW) of $19.4 \pm 3.4$ (range: 18.6-21.2) kg, were used as experimental animals. Only male pigs were included in the current study to avoid the variability induced by sexual hormones. All animals were anesthetized by intramuscular injection of xylazine hydrochloride $(0.3 \mathrm{ml} / \mathrm{kg} \mathrm{BW}$, Shengda Animal Medicine Co., Ltd., Shengda, China) combined with pentobarbital sodium (20 mg/kg BW, Merck Serono KGaA, Darmstadt, Germany).

After the surgical sites were shaved and sterilized, the right rear limbs (as the Ba group) were operated at approximately $10.0 \mathrm{~cm}$ from the patella to the tibial tubercle by using a lateral parapatellar approach. The joint was opened partly by medial patella luxation. The ACL was fixed by a clamp and was cut by $1.0 \mathrm{~cm}$ at the distal end, as described previously ${ }^{11}$. The LCL was separated and exposed along the joint line and resected for a length of $1.0 \mathrm{~cm}$. After washing with $100 \mathrm{ml}$ of sterile saline, the articular capsule was sutured intermittently and independently with 3-0 silk sutures (Aipu Medical Equipment Co., Ltd., Hangzhou, China). The operation was carried out on the left rear limbs as the sham group (Aa group) as similar to the above incision of the articular capsule, but no manipulation was made to the ligaments, menisci, cartilage, or bone. There was no randomization regarding the grouping of the rear limbs to allow for easy recognition of the experimental and control limbs after the operation.

The mini-pigs were checked twice a day after the operation. All animals were treated with penicillin (Kerui Animal Pharmaceutical Co., Ltd, Chengdu, China) and tramadol (Duoduo Pharmaceutical Co., Ltd, Jiamusi, China) for 7 days to avoid infections. After the wound was completely healed, the animal could move freely in the pigpen.

Preparation procedure of meniscal tissue. The animals were sacrificed at 26 weeks after surgery by acute massive exsanguination. The menisci were isolated using a standardized preparation technique. After the knee joint was opened from the front, the joint capsule and the ligaments were cut along the joint line near the side of the femur to separate the tibia from the femur. The medial menisci were carefully removed in one piece from the tibia. They were washed with phosphate-buffered saline (PBS) and cut into soybean-sized pieces. The preparation was carried out without touching the surfaces of the menisci or the hyaline articular cartilage to prevent contamination and destruction of the meniscal surface and deep structures. The samples were taken from the red-white zone of the meniscal pars intermedia, followed by placing in a cryopreservation tube for immediate freezing and storing in liquid nitrogen. The sampling was performed immediately after the death of the mini-pigs and was completed within $10 \mathrm{~min}$. All equipment was treated with hydrogen peroxide to remove eventual RNAse.

RNA extraction and assessment. After DNase digestion, the total RNA from the meniscal tissue was extracted using an E.Z.N.A Total RNA Kit II (Omega Bio-Tek Inc., Norcross, GA, USA) according to the manufacturer's instructions. The quantity and quality of RNA were measured using a NanoDrop ND-1000 spectrophotometer (Thermo Fisher Scientific, Waltham, MA, USA). RNA integrity was assessed by standard denaturing agarose gel electrophoresis.

Agilent microarray study. RNA labeling and array hybridization were performed according to the Agilent One-Color Microarray-Based Gene Expression Analysis protocol (Agilent Technologies, Santa Clara, CA, USA). Briefly, the total RNA from each sample was linearly amplified and labeled with Cy3-UTP using the Agilent Quick Amp One-Color labeling kit (Agilent Technologies, Santa Clara, CA, USA). The Labeled cRNAs were purified using the RNeasy Mini Kit (Qiagen, Venlo, The Netherlands) according to the manufacturer's instructions. The concentration and specific activity of the labeled cRNAs (pmol Cy3/ $\mu \mathrm{g}$ cRNA) were measured using a NanoDrop ND-1000 spectrophotometer (Thermo Fisher Scientific, Waltham, MA, USA). After that, $1 \mu \mathrm{g}$ of each labeled cRNA was fragmented by adding $11 \mu \mathrm{l}$ of $10 \times$ blocking agent and $2.2 \mu \mathrm{l}$ of $25 \times$ fragmentation buffer and heating the mixture at $60^{\circ} \mathrm{C}$ for $30 \mathrm{~min}$. Finally, $55 \mu \mathrm{l}$ of $2 \times \mathrm{GE}$ hybridization buffer was added to dilute the labeled cRNA. Then, $100 \mu$ of the hybridization solution was dispensed into the gasket slide and assembled to the gene expression microarray slide. The slides were incubated for $17 \mathrm{~h}$ at $65^{\circ} \mathrm{C}$ in a hybridization oven (Agilent Technologies, Santa Clara, CA, USA). The RNA was hybridized to the Whole Pig Genome Microarray (Agilent Technologies, Santa Clara, CA, USA). The hybridized arrays were washed, fixed, and scanned using a DNA Microarray Scanner (G2505C, Agilent Technologies, Santa Clara, CA, USA) ${ }^{12}$.

Microarray data analyses. The raw gene expression data were extracted from the array images using the Feature Extraction software (version 11.0.1.1, Agilent Technologies, Santa Clara, CA, USA). Quantile normalization and subsequent data processing were performed with the GeneSpring GX v12.1 software package (Agilent Technologies, Santa Clara, CA, USA). After quantile normalization of the raw data, the genes from at least seven out of the 14 samples had flags detected ("All Targets Value") and were chosen for further data analysis. Statistically significant differentially expressed genes (DEGs) between the two groups were identified through volcano plot filtering, using a threshold of fold-change $>2.0$ and $\mathrm{P}$-value $<0.05$ based on the Student t-test in the GeneSpring software). DEGs between the two samples were identified through fold change filtering. Unsupervised hierarchical clustering was performed using the $\mathrm{R}$ scripts to compare gene expression profiles among the 
samples of the same group. Gene ontology (GO, http://geneontology.org) analysis and pathway analysis were performed with the standard enrichment computation method to associate the DEGs with GO categories and pathways. The GO categories comprised of three structured networks (biological processes, cellular components, and molecular function) of defined terms to describe the gene functions. To perform the GO analysis of differential genes, top GO was used to infer the molecular function they are involved in ${ }^{12}$. The KEGG pathway analysis was performed to infer the pathways the DEGs are involved in ${ }^{13-15}$. All the microarray data are MIAME compliant, and the raw data are available through the GEO database with the accession number GSE145402.

Real-time RT-PCR analysis. Twelve genes were selected for validation. The quantification of transcript levels for the selected genes and the housekeeping gene GAPDH was performed using the ViiA 7 Real-time PCR System (Applied Biosystems, Foster City, CA, USA). PCR primers were designed based on cDNA sequences from the NCBI Sequence database using Primer 5.0 (PREMIER Biosoft International, Palo Alto, CA, USA). The Gene Amp PCR System 9700 (Applied Biosystems, Foster City, CA, USA) was used to generate the first-strand cDNA from the isolated RNA. The cDNA was amplified with an initial Taq DNA polymerase activation step at $95^{\circ} \mathrm{C}$ for $10 \mathrm{~min}$, followed by 40 cycles of denaturation at $95^{\circ} \mathrm{C}$ for $10 \mathrm{~s}$ and annealing at $60^{\circ} \mathrm{C}$ for $60 \mathrm{~s}$, according to the manufacturer's instructions. Each reaction was repeated three times, and the Ct values were obtained in triplicates for each gene. The expression level of the selected genes was normalized to GAPDH using the $2^{-\Delta \Delta \mathrm{Ct}}$ method. Each real-time RT-PCR experiment was repeated twice in triplicate. Statistical significance of the differences between the two groups was determined by the Student $\mathrm{t}$-test while $\mathrm{P}<0.05$, using SPSS 19.0 for Windows (IBM, Armonk, NY, USA).

Ethical approval. All procedures were reviewed and approved by the Medical Ethics Committee of Hainan General Hospital (Med-Eth-Re [2020] 5). All experiments were performed in accordance with the relevant guidelines and regulations.

\section{Results}

DEG identification. The volcano map of all the data obtained after the chip scan can easily and reasonably reflect the distribution of the DEGs between the two groups (Fig. 1). According to the DEG criteria of fold-change $>2$ and P-value $<0.05,893$ DEGs were found, among which 537 are upregulated and 356 are downregulated. The top ten upregulated DEGs include TFAP2D, HOXD13, CES1, PAK5, C7H6orf15, GCNT7, VIL1, COL7A1, CD81, and SLC7A8 (Table 1). The top ten down-regulated DEGs include SPMI, INA, CPS1, PGA5, LYRM4, TMCO5A, LOC100520832, CFAP58, ESRP1, and VMA21 (Table 2).

Unsupervised hierarchical clustering. The unsupervised cluster analysis of DEGs obtained after comparison of the two groups demonstrate the relationship as well as the difference between the groups. The results show that the two groups are well separated in terms of the DEGs (Fig. 2). All samples are accurately clustered into the same cluster of the corresponding category, indicating the reliability of the results of chip analysis, and that the gene expression pattern of the experimental group in comparison to the control group is consistent.

Results of the GO enrichment analysis. The DEGs obtained from the above analysis were classified into biological processes, cellular components, and molecular functions according to the functional relevance of the genes. The top ten biological processes with upregulated gene enrichment scores included the cellular response to hormone stimulus, response to hormones, cellular response to endogenous stimulus, C21-steroid hormone metabolic process, neuropeptide signaling pathway, negative regulation of reactive oxygen species metabolic process, regulation of nitric oxide biosynthetic process, response to endogenous stimulus, nitric oxide biosynthetic process, and nitric oxide metabolic process (Table 3 and Fig. $3 \mathrm{~A}$ ).

The top ten biological processes with down-regulated gene enrichment scores included sex determination, muscle fiber development, mesenchymal morphogenesis, negative regulation of the reproductive process, male gonad development, primary male sexual characteristics development, striped muscle cell development, male sex differentiation, muscle cell development, and reproductive structure development (Table 4 and Fig. 3B).

The cellular components involved with upregulated DEGs included transcription factor complex, an intrinsic component of membrane, cell-substrate adherens junction, and cell-substrate junction (Table 5 and Fig. 4A). Cellular components involved with down-regulated DEGs included sarcomere, contractile fiber part, myofibril, contractile fiber, cell body, integral component of the plasma membrane, endoplasmic reticulum membrane, nuclear outer membrane-endoplasmic reticulum membrane network, endoplasmic reticulum subcompartment, intrinsic component of the plasma membrane, plasma membrane part, and endoplasmic reticulum part (Table 6 and Fig. 4B).

The top ten molecular functions with upregulated gene enrichment scores included transcription regulatory region sequence-specific DNA binding, sequence-specific double-stranded DNA binding, virus receptor activity, hijacked molecular function, transition metal ion binding, iron ion binding, double-stranded DNA binding, regulatory region nucleic acid binding, transcription regulatory region DNA binding, and RNA polymerase II regulatory region sequence-specific DNA binding (Table 7 and Fig. 5A). The molecular functions involved with downregulated DEGs included transcription factor activity, RNA polymerase II distal enhancer sequence-specific binding, horizon activity, enhancer-binding, DNA binding transcription factor activity, transcription regulator activity, and neuropeptide binding (Table 8 and Fig. 5B). 


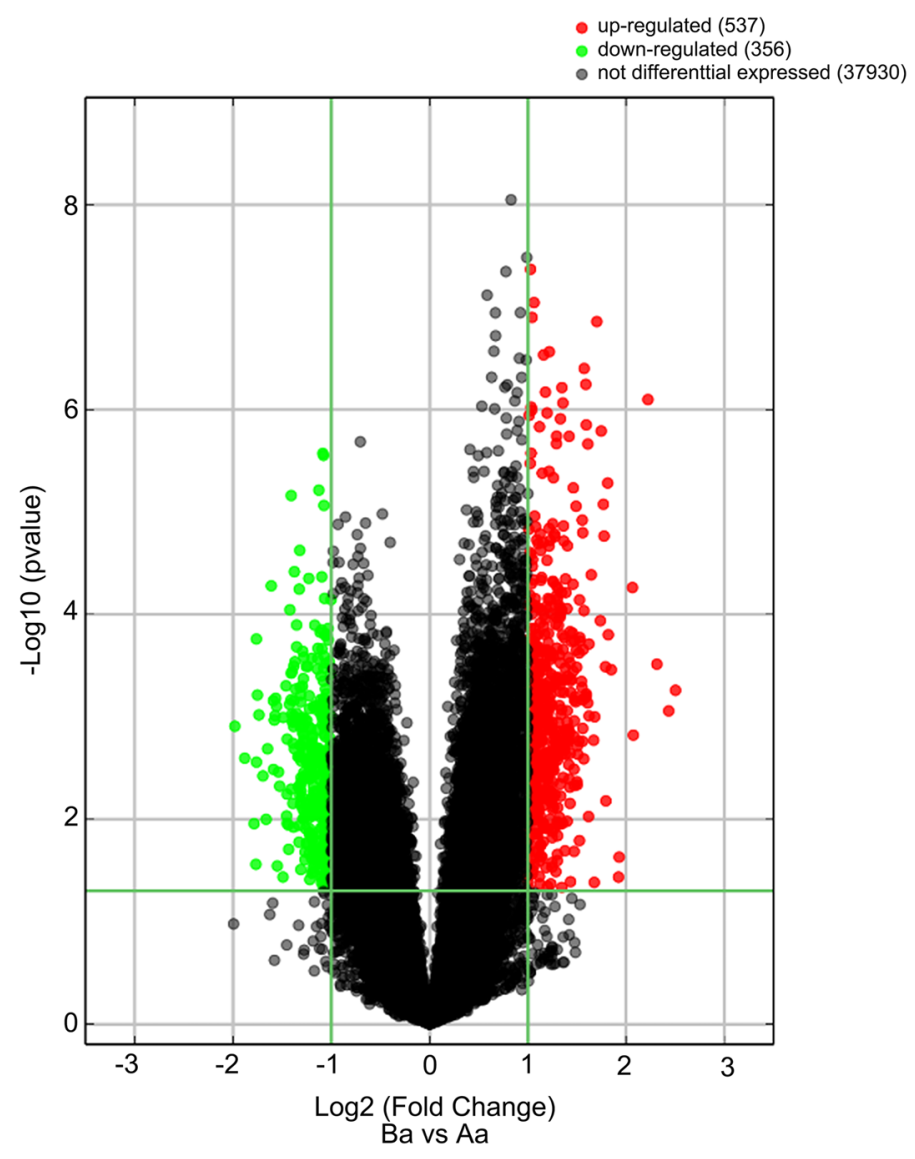

Figure 1. The volcano plot map of all the genes. Differentially expressed genes (DEGs) were determined while fold-change $>2$ and P-value $<0.05 .893$ DEGs were found, among which 537 are upregulated and 356 are downregulated.

\begin{tabular}{|l|l|l|l|l|l|}
\hline Genbank accession & Gene symbol & Description & P-value & FDR & Fold change \\
\hline XM_003356639 & TFAP2D & Transcription factor AP-2 delta & 0.000551587 & 0.023345329 & 5.6602732 \\
\hline XM_003483680 & HOXD13 & Homeobox D13 & 0.000877072 & 0.028532155 & 5.3957126 \\
\hline NM_214246 & CES1 & Sus scrofa carboxylesterase 1 (CES1) & $5.4688 \mathrm{E}-05$ & 0.008853756 & 4.1784836 \\
\hline AK393241 & PAK5 & $\begin{array}{l}\text { Rep: Serine/threonine-protein kinase PAK 7-Mus } \\
\text { musculus (Mouse), partial (21\%) }\end{array}$ & $1.72044 \mathrm{E}-05$ & 0.00514212 & 3.4211194 \\
\hline NM_001128447 & C7H6orf15 & $\begin{array}{l}\text { Sus scrofa chromosome 7 open reading frame, } \\
\text { human C6orf15 (C7H6orf15) }\end{array}$ & $8.41952 \mathrm{E}-06$ & 0.003760236 & 3.4049223 \\
\hline NM_001113699 & GCNT7 & $\begin{array}{l}\text { Sus scrofa glucosaminyl (N-acetyl) transferase fam- } \\
\text { ily member 7 (GCNT7) }\end{array}$ & $1.38326 \mathrm{E}-07$ & 0.000537464 & 3.2506906 \\
\hline AK231343 & VIL1 & $\begin{array}{l}\text { Rep: cyclic peptide transporter precursor-Methy- } \\
\text { lobacteriumextorquens PA1, partial (4\%) }\end{array}$ & 0.001001336 & 0.030467417 & 3.2032195 \\
\hline XM_005669519 & COL7A1 & Collagen type VII alpha 1 chain & 0.000982785 & 0.030115232 & 3.0775177 \\
\hline BX670110 & CD81 & $\begin{array}{l}\text { Sus Scrofa library (scac) Sus scrofa cDNA clone } \\
\text { scac0034.n.11 3prim }\end{array}$ & $1.4119 \mathrm{E}-06$ & 0.001443671 & 3.0181273 \\
\hline XM_003128550 & SLC7A8 & Solute carrier family 7 member 8 & $7.2232 \mathrm{E}-05$ & 0.010036037 & 2.8769457 \\
\hline
\end{tabular}

Table 1. The top ten differential genes with up-regulation.

Results of the pathway analysis. According to the DEGs, a total of 36 signaling pathways with differential regulation were found. Among these, the top ten pathways included type II diabetes mellitus, taste transduction, prolactin signaling pathway, longevity regulating pathway, ovarian steroidogenesis, neuroactive ligandreceptor interaction, inflammatory mediator regulation of TRP channels, pantothenate and CoA biosynthesis, AMPK signaling pathway, and bile secretion. The down-regulated signaling pathways included thyroid hormone synthesis, cocaine addiction, metabolism of xenobiotics by cytochrome P450, legionellosis, glycerolipid metabo- 
www.nature.com/scientificreports/

\begin{tabular}{|c|c|c|c|c|c|}
\hline Genbank accession & Gene symbol & Description & P-value & FDR & Fold change \\
\hline NM_001031776 & $S P M I$ & $\begin{array}{l}\text { Sus scrofa seminal plasma sperm motility inhibitor/spermadhesin AQN-3-like protein } \\
\text { (SPMI) }\end{array}$ & 0.002536463 & 0.047702946 & 3.6789604 \\
\hline XM_001929320 & INA & Internexin neuronal intermediate filament protein alpha & 0.002054744 & 0.042969359 & 3.1323546 \\
\hline AK394043 & CPS1 & Carbamoyl-phosphate synthase 1 & $5.26505 \mathrm{E}-05$ & 0.008631794 & 3.0512209 \\
\hline NM_213873 & PGA5 & Sus scrofa pepsinogen 5, group I (pepsinogen A) (PGA5) & 0.003462281 & 0.056295579 & 2.9000267 \\
\hline AK233279 & LYRM4 & Ribonuclease P/MRP subunit p40 & 0.036770923 & 0.197230012 & 2.8101604 \\
\hline XM_001927899 & TMCO5A & Transmembrane and coiled-coil domains 5A & 0.009299658 & 0.095443941 & 2.7383115 \\
\hline NM_001285972 & LOC100520832 & Sus scrofalithostathine-like (LOC100520832) & 0.005710985 & 0.073428296 & 2.7296819 \\
\hline XM_005671443 & CFAP58 & Cilia and flagella associated protein 58 & 0.019700007 & 0.140371132 & 2.6994439 \\
\hline AK396999 & ESRP1 & Epithelial splicing regulatory protein 1 & 0.000755832 & 0.026771077 & 2.6832393 \\
\hline AK343969 & VMA21 & VMA21, vacuolar ATPase assembly factor & 0.000363673 & 0.01979061 & 2.6355477 \\
\hline
\end{tabular}

Table 2. The top ten differential genes with down-regulation.

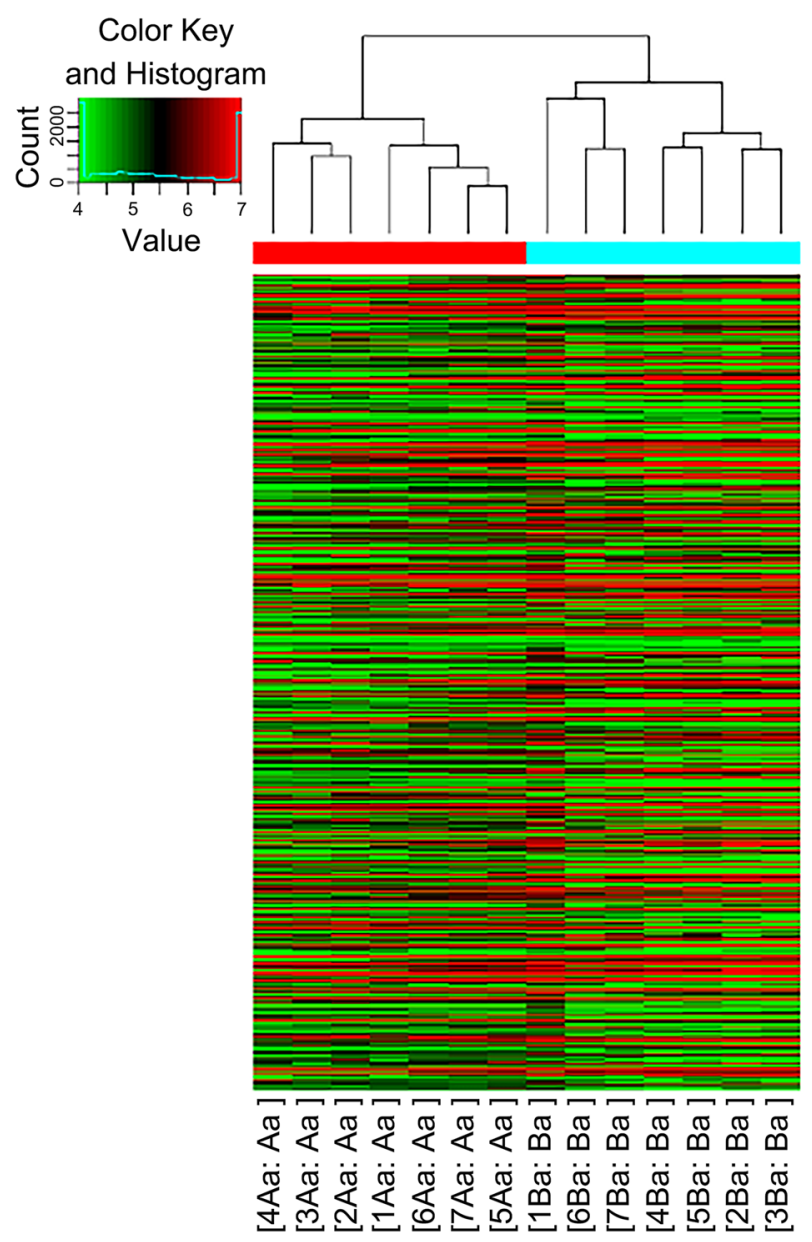

Figure 2. The dendrogram. The meniscal degeneration group is shown in cyan blue, while the control group is shown in red.

lism, chemical carcinogenesis, amphetamine addiction, acute myeloid leukemia, adherens junction, Salmonella infection, protein digestion and absorption, and hematopoietic cell lineage (Tables 9 and 10 and Fig. 6).

PCR validation of DEGs. According to the microarray analysis results, 12 DEGs were selected for validation by real-time quantitative PCR. The selected DEGs are involved in nitric oxide metabolic process (PRL and ACP5), male gonad development (DMRT1), muscle fiber development (ACTA1), transition metal ion binding and metal ion binding (CYP17A1 and ACP5), integral component of the plasma membrane (SSTR2), endoplasmic reticulum membrane (EPHX1), type II diabetes mellitus pathway (ABCC8, ADIPOQ, and SLC2A4), 


\begin{tabular}{|c|c|c|c|c|}
\hline GO.ID & Term & P value & Enrichment_Score & Genes \\
\hline GO:0032870 & Cellular response to hormone stimulus & 0.00031193 & 3.505944124 & $\begin{array}{l}\text { NR5A1//SLC2A4//ADIPOR2//SOCS2//P2RY4//MYOD1// } \\
\text { HCRTR2 }\end{array}$ \\
\hline GO:0009725 & Response to hormone & 0.00115203 & 2.938536525 & $\begin{array}{l}\text { NR5A1//SLC2A4//HCRTR2//ADIPOR2//SOCS2//P2RY4// } \\
\text { MYOD1 }\end{array}$ \\
\hline GO:0071495 & Cellular response to endogenous stimulus & 0.00356694 & 2.447703824 & $\begin{array}{l}\text { NR5A1//SLC2A4//HCRTR2//ADIPOR2//SOCS2//P2RY4// } \\
\text { MYOD1//FGF21 }\end{array}$ \\
\hline GO:0008207 & C21-steroid hormone metabolic process & 0.00358808 & 2.445137806 & CYP17A1//PRL \\
\hline GO:0007218 & Neuropeptide signaling pathway & 0.00394089 & 2.404406092 & SCG5//NPY2R//HCRTR2 \\
\hline GO:2000378 & $\begin{array}{l}\text { Negative regulation of reactive oxygen species metabolic } \\
\text { process }\end{array}$ & 0.00435963 & 2.360550537 & $P R L / / A C P 5$ \\
\hline GO:0045428 & Regulation of nitric oxide biosynthetic process & 0.00708678 & 2.149550997 & PRL//ACP5 \\
\hline GO:0009719 & Response to endogenous stimulus & 0.00714517 & 2.14598759 & $\begin{array}{l}\text { NR5A1//SLC2A4//HCRTR2//ADIPOR2//SOCS2//P2RY4// } \\
\text { MYOD1//FGF21 }\end{array}$ \\
\hline GO:0006809 & Nitric oxide biosynthetic process & 0.00923586 & 2.034522585 & PRL//ACP5 \\
\hline GO:0046209 & Nitric oxide metabolic process & 0.00923586 & 2.034522585 & PRL//ACP5 \\
\hline GO:2001057 & Reactive nitrogen species metabolic process & 0.00923586 & 2.034522585 & PRL//ACP5 \\
\hline GO:0048732 & Gland development & 0.01028441 & 1.987820429 & PRL//CSN2//NR5A1//HOXD13 \\
\hline GO:0042445 & Hormone metabolic process & 0.01130856 & 1.946592525 & CYP17A1//PRL//NR5A1 \\
\hline GO:1903426 & Regulation of reactive oxygen species biosynthetic process & 0.01163811 & 1.934117409 & PRL//ACP5 \\
\hline GO:0007589 & Body fluid secretion & 0.01293115 & 1.888362813 & PRL//CSN2 \\
\hline GO:0042446 & Hormone biosynthetic process & 0.01569514 & 1.804234938 & CYP17A1//PRL \\
\hline GO:0034754 & Cellular hormone metabolic process & 0.01868863 & 1.728422521 & CYP17A1//PRL \\
\hline GO:1903409 & Reactive oxygen species biosynthetic process & 0.02026862 & 1.693175919 & PRL//ACP5 \\
\hline GO:0042136 & Neurotransmitter biosynthetic process & 0.02190261 & 1.65950407 & PRL//ACP5 \\
\hline GO:0007631 & Feeding behavior & 0.02358953 & 1.627280649 & NPY2R//HCRTR2 \\
\hline GO:0042221 & Response to chemical & 0.02361703 & 1.626774706 & $\begin{array}{l}\text { PTK2B//S100A12//CCR10//NR5A1//SOCS2//SLC52A2// } \\
\text { ACP5//SLC2A4//HCRTR2//ADIPOR2//P2RY4//MYOD1// } \\
\text { PRL//FGF21 }\end{array}$ \\
\hline GO:0070887 & Cellular response to chemical stimulus & 0.0238929 & 1.621731065 & $\begin{array}{l}\text { PTK2B//S100A12//NR5A1//SOCS2//SLC2A4//HCRTR2//ADI- } \\
\text { POR2//P2RY4//MYOD1//PRL//FGF21 }\end{array}$ \\
\hline GO:0046849 & Bone remodeling & 0.02711786 & 1.566744651 & ACP5//SPP2 \\
\hline GO:0030879 & Mammary gland development & 0.03679131 & 1.434254721 & PRL//CSN2 \\
\hline GO:1901700 & Response to oxygen-containing compound & 0.04136472 & 1.383369859 & ACP5//SLC2A4//SOCS2//P2RY4//MYOD1//HCRTR2 \\
\hline GO:0009755 & Hormone-mediated signaling pathway & 0.04314004 & 1.365119451 & ADIPOR2//NR5A1 \\
\hline GO:0042133 & Neurotransmitter metabolic process & 0.04314004 & 1.365119451 & PRL//ACP5 \\
\hline GO:2000377 & Regulation of reactive oxygen species metabolic process & 0.04314004 & 1.365119451 & PRL//ACP5 \\
\hline GO:1901652 & Response to peptide & 0.04836282 & 1.315488391 & SLC2A4//SOCS2//HCRTR2 \\
\hline
\end{tabular}

Table 3. The biological processes with up-regulated gene enrichment scores.

A

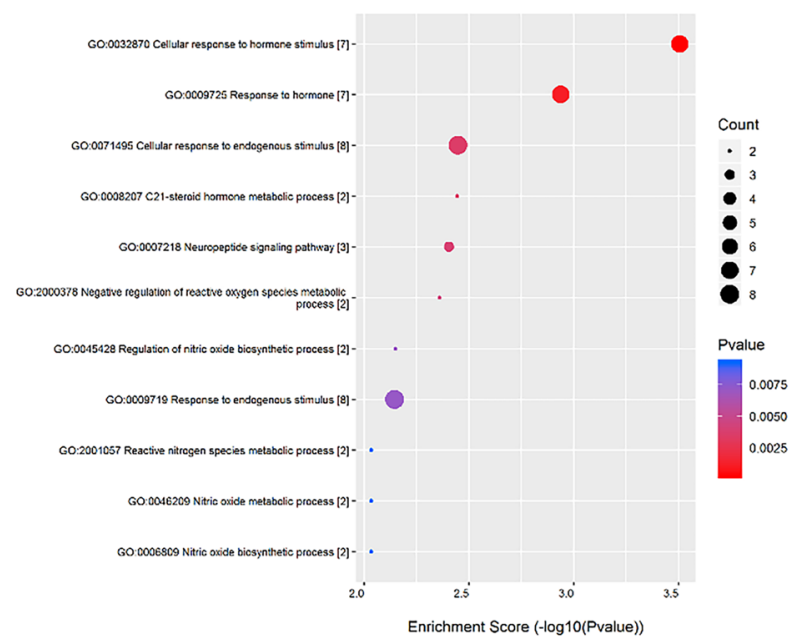

B

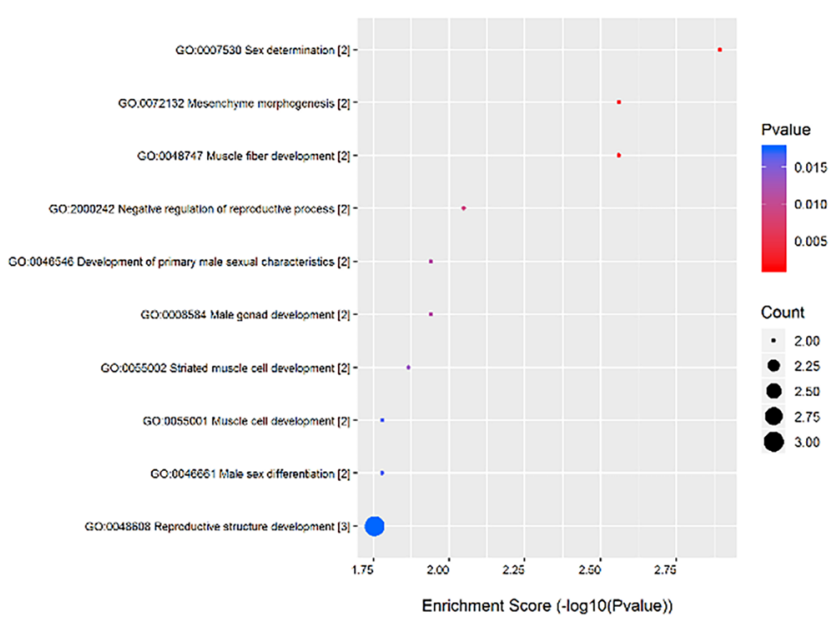

Figure 3. The biological processes enrichment analyses for the differentially expressed genes (DEGs) analysed by Gene Ontology (GO). (A) The top ten biological processes with upregulated Enrichment Score Dot Plot. (B) The top ten biological processes with down-regulated Enrichment Score Dot Plot. 


\begin{tabular}{|c|c|c|c|c|}
\hline GO.ID & Term & P value & Enrichment_Score & Genes \\
\hline GO:0007530 & Sex determination & 0.00128145 & 2.89230002 & DMRT1//NR5A1 \\
\hline GO:0048747 & Muscle fiber development & 0.00275321 & 2.560160546 & ACTA1//LEF1 \\
\hline GO:0072132 & Mesenchyme morphogenesis & 0.00275321 & 2.560160546 & LEF1//ACTA1 \\
\hline GO:2000242 & $\begin{array}{l}\text { Negative regulation of reproductive } \\
\text { process }\end{array}$ & 0.00895073 & 2.048141367 & DMRT1//NR5A1 \\
\hline GO:0008584 & Male gonad development & 0.01149866 & 1.939352658 & DMRT1//NR5A1 \\
\hline GO:0046546 & $\begin{array}{l}\text { Development of primary male sexual } \\
\text { characteristics }\end{array}$ & 0.01149866 & 1.939352658 & NR5A1//DMRT1 \\
\hline GO:0055002 & Striated muscle cell development & 0.01359466 & 1.866631736 & ACTA1//LEF1 \\
\hline GO:0046661 & Male sex differentiation & 0.01662697 & 1.779187017 & NR5A1//DMRT1 \\
\hline GO:0055001 & Muscle cell development & 0.01662697 & 1.779187017 & ACTA1//LEF1 \\
\hline GO:0048608 & Reproductive structure development & 0.0175686 & 1.755262805 & NR5A1//DMRT1//LEF1 \\
\hline GO:0061458 & Reproductive system development & 0.0179883 & 1.745009826 & NR5A1//DMRT1//LEF1 \\
\hline GO:0010817 & Regulation of hormone levels & 0.01884459 & 1.724813227 & NR5A1//TTR//SSTR2 \\
\hline GO:0030855 & Epithelial cell differentiation & 0.02062492 & 1.685607828 & DMRT1//LEF1//UPK2 \\
\hline GO:0042445 & Hormone metabolic process & 0.02628193 & 1.580342705 & TTR//NR5A1 \\
\hline GO:0051146 & Striated muscle cell differentiation & 0.0333557 & 1.476829929 & ACTA1//LEF1 \\
\hline GO:0009888 & Tissue development & 0.03500311 & 1.455893416 & LEF1//UPK2//ACTA1//DMRT1//NR5A1 \\
\hline GO:0008406 & Gonad development & 0.03550021 & 1.449769058 & NR5A1//DMRT1 \\
\hline GO:0045137 & $\begin{array}{l}\text { Development of primary sexual character- } \\
\text { istics }\end{array}$ & 0.03550021 & 1.449769058 & NR5A1//DMRT1 \\
\hline GO:0060485 & Mesenchymal development & 0.03881534 & 1.410996639 & LEF1//ACTA1 \\
\hline GO:2000241 & Regulation of reproductive process & 0.03881534 & 1.410996639 & DMRT1//NR5A1 \\
\hline GO:0007517 & Muscle organ development & 0.04108929 & 1.386271408 & LEF1//ACTA1 \\
\hline GO:0010171 & Body morphogenesis & 0.04880594 & 1.311527279 & LEF1 \\
\hline GO:0021879 & Forebrain neuron differentiation & 0.04880594 & 1.311527279 & LEF1 \\
\hline GO:0032673 & Regulation of interleukin-4 production & 0.04880594 & 1.311527279 & LEF1 \\
\hline GO:0045840 & $\begin{array}{l}\text { Positive regulation of mitotic nuclear } \\
\text { division }\end{array}$ & 0.04880594 & 1.311527279 & DMRT1 \\
\hline GO:0060713 & Labyrinthine layer morphogenesis & 0.04880594 & 1.311527279 & LEF1 \\
\hline
\end{tabular}

Table 4. The biological processes with down-regulated gene enrichment scores.

\begin{tabular}{|l|l|l|l|l|}
\hline GO.ID & Term & P value & Enrichment_Score & Genes \\
\hline GO:0005667 & Transcription factor complex & 0.01480675 & 1.829540202 & NR5A1//GTF2B//MYOD1//TFAP2D \\
\hline GO:0031224 & Intrinsic component of membrane & 0.03410257 & 1.467212853 & $\begin{array}{l}\text { SLC2A4//TMPRSS15//IL2RG//IGF2R//NPY2R//CYP2C33//CYP4A21//ADI- } \\
\text { POR2//P2RY4//CCR10//CD81//MUC13//GCNT7//GABBR1//ACE2//DLK2// } \\
\text { HRK//LOC100156225//ADAM30//IGSF1//CLDN6//SYNDIG1L//LOC100520032// } \\
\text { LOC100520992//CWH43//PAQR5//RXFP1//VNN1//HCRTR2//SLC52A2//TSPAN1// } \\
\text { SLC25A44//DLG3 }\end{array}$ \\
\hline GO:0005924 & Cell-substrate adherens junction & 0.04360798 & 1.360434073 & PTK2B//SMPX \\
\hline GO:0030055 & Cell-substrate junction & 0.04360798 & 1.360434073 & PTK2B//SMPX \\
\hline
\end{tabular}

Table 5. The Cellular components with up-regulated gene enrichment scores.

inflammatory mediator regulation of TRP channels pathway (ADCY4 and TRPA1), and AMPK signaling pathway (ADIPOQ and SLC2A4). The results are shown in Table 11 and Fig. 7. The results indicate that the microarray data accurately reflects the gene expression patterns.

\section{Discussion}

The insufficiency of the ACL can lead to meniscal damage ${ }^{16}$. A previous study of meniscal degeneration models in mini-pigs showed that removing the ACL leads to meniscal degeneration changes as observed by histology ${ }^{11}$. In addition, the real-time quantitative PCR analysis showed that COL1A1 is highly expressed in the degenerative meniscus ${ }^{11}$. In this study, the meniscus degeneration model was constructed by removing the ACL and LCL, based on the Pond-Nuki model ${ }^{10}$. The function of the meniscus is mainly to increase knee stability, absorb shocks, and conduct loads. When other stabilizing structures of the knee joint (such as the ligaments) are impaired, the contribution of the meniscus is increased, leading to stress and wear. This stress is mainly manifested in the horizontal shear plane and is the main cause of meniscal tear after ACL rupture. In addition, after LCL resection, the knee joint shows a loose lateral stable structure, causing varus change of the knee and shifting the rear limb force line inward. More stress is then taken by the medial meniscus, which can reach $60-80 \%$ of the $\mathrm{BW}^{17}$. The 


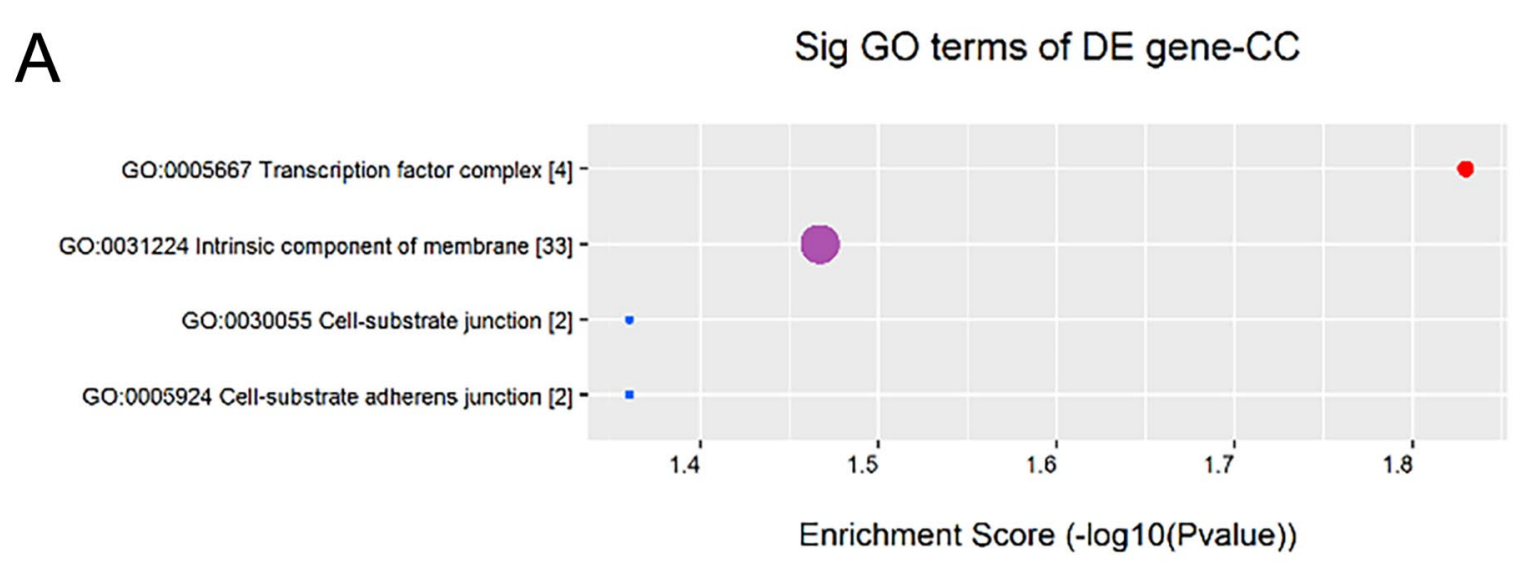

\section{Sig GO terms of DE gene-CC}

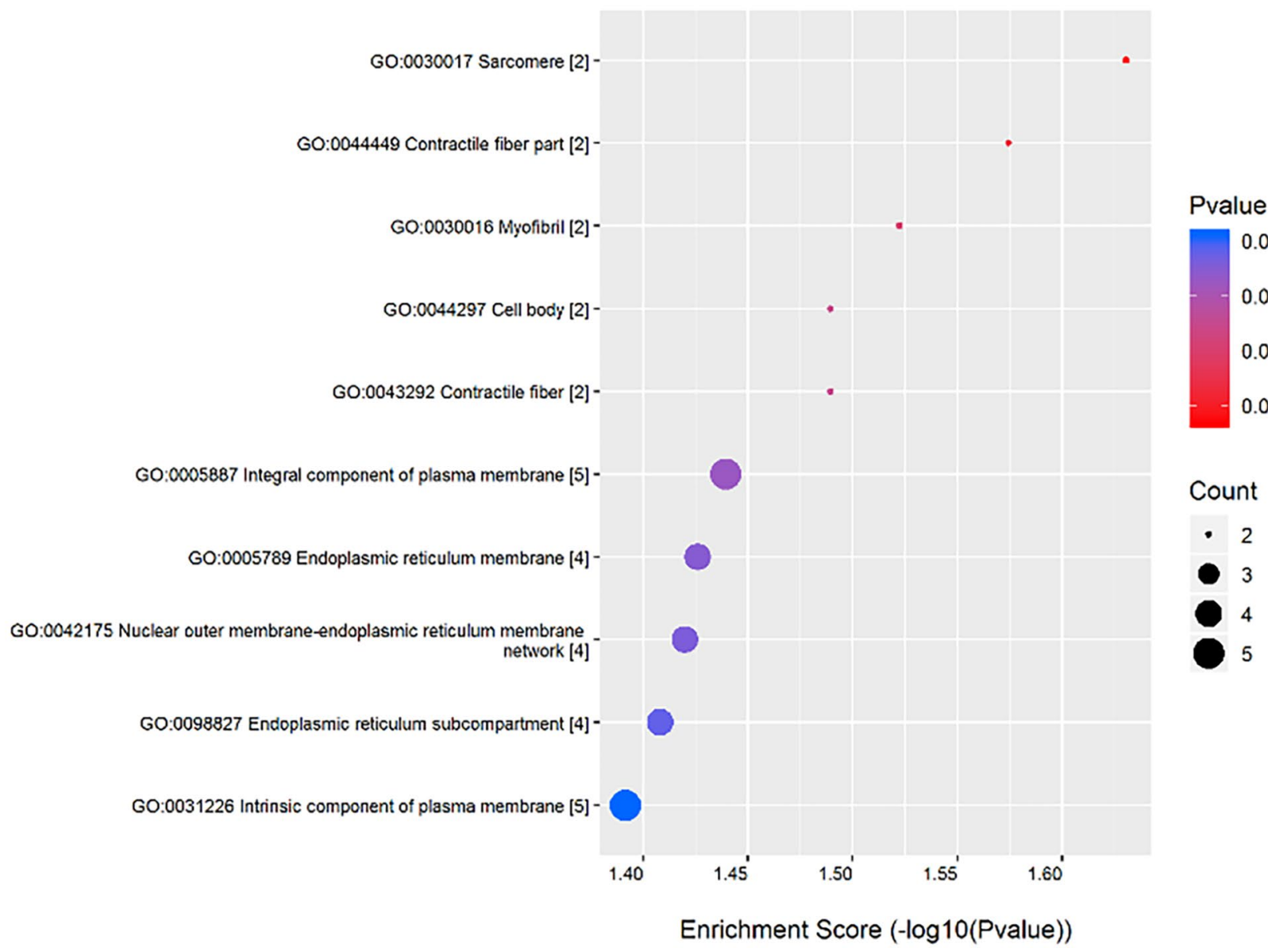

Figure 4. The cellular components enrichment analyses for the differentially expressed genes (DEGs) analysed by Gene Ontology (GO). (A) The Cellular components with upregulated Enrichment Score Dot Plot. (B) The Cellular components with down-regulated Enrichment Score Dot Plot. 


\begin{tabular}{|c|c|c|c|c|}
\hline GO.ID & Term & P value & Enrichment_Score & Genes \\
\hline GO:0030017 & Sarcomere & 0.02341886 & 1.630434303 & ACTA1//SMPX \\
\hline GO:0044449 & Contractile fiber part & 0.02664338 & 1.574410611 & SMPX//ACTA1 \\
\hline GO:0030016 & Myofibril & 0.03003724 & 1.522340041 & SMPX//ACTA1 \\
\hline GO:0043292 & Contractile fiber & 0.03239074 & 1.489579145 & SMPX//ACTA1 \\
\hline GO:0044297 & Cell body & 0.03239074 & 1.489579145 & PDYN//ACTA1 \\
\hline GO:0005887 & Integral component of plasma membrane & 0.03635516 & 1.439433886 & SSTR2//SLC12A5//CASR//HTR2C//ACKR2 \\
\hline GO:0005789 & Endoplasmic reticulum membrane & 0.03748493 & 1.426143298 & EPHX1//CYP2E1//RTN4//VMA21 \\
\hline GO:0042175 & $\begin{array}{l}\text { Nuclear outer membrane-endoplasmic reticulum membrane } \\
\text { network }\end{array}$ & 0.03800801 & 1.42012482 & EPHX1//CYP2E1//RTN4//VMA21 \\
\hline GO:0098827 & Endoplasmic reticulum subcompartment & 0.03906695 & 1.408190446 & EPHX1//CYP2E1//RTN4//VMA21 \\
\hline GO:0031226 & Intrinsic component of plasma membrane & 0.04059138 & 1.39156617 & SLC12A5//CASR//HTR2C//ACKR2//SSTR2 \\
\hline GO:0044459 & Plasma membrane part & 0.04118319 & 1.385280052 & SLC12A5//CASR//HTR2C//ACKR2//UPK2//SGCZ//SSTR2 \\
\hline GO:0044432 & Endoplasmic reticulum part & 0.04936582 & 1.306573638 & EPHX1//CYP2E1//RTN4//VMA21 \\
\hline
\end{tabular}

Table 6. The Cellular components with down-regulated gene enrichment scores.

advantage of the mini-pig model over smaller animals is that the degree of the load is close to that of the human characteristics, but the disadvantages include longer modeling time and higher animal facility requirements ${ }^{5}$.

The results of the DEG cluster analysis showed that all samples could be accurately aggregated into the right grouping, indicating that the experimental intervention induced the DEGs. This is consistent with other studies that showed that ACL resection induces morphological, histological, and genetic changes in the meniscus ${ }^{11,18}$. A study in humans showed that changes in DEGs occur in ligaments after injury and that such changes might be observed in other musculoskeletal tissues ${ }^{19}$.

Meniscal stress is associated with inflammation. One of the most important mediators in inflammation is nitric oxide, wherein it upregulates meniscus matrix catabolism and pro-inflammatory gene expression, resulting in increased meniscus glycosaminoglycan release and meniscal impairment ${ }^{20,21}$. Nitric oxide can reduce autophagy by down-regulating the JNK signaling pathway, thereby affecting meniscal repair and causing meniscal degeneration $^{22}$. Hyaluronic acid, selenium, and interleukin-10 delay or improve meniscus degeneration due to the inhibition of nitric oxide ${ }^{23-25}$. This study showed that the upregulated biological processes included regulation of nitric oxide biosynthesis process, nitric oxide biosynthetic process, and nitric oxide metabolic process, indicating that nitric oxide plays an important role in meniscal degeneration.

There is a clear sex difference in knee osteoarthritis and meniscus degeneration ${ }^{26-28}$, suggesting the role of sex hormones in meniscal degeneration. Although our study used male Wuzhishan mini-pigs, many male-associated biological processes were down-regulated in the DEG analysis, such as sex determination, negative regulation of the reproductive process, male gonadal development, primary male sexual characteristics development, male sex differentiation, and reproductive structure development. This suggests that the main feature of male characteristics are down-regulated by meniscal degeneration, indicating that androgens might play a role in promoting meniscus damage repair, while its decreased expression level promotes meniscal degeneration. Secondly, sex hormones might indirectly cause meniscal degeneration by affecting muscle strength, walking endurance, and balance ability ${ }^{29}$. Indeed, androgens promote the development of the skeletal muscle system ${ }^{30,31}$. When this dynamic stabilization mechanism decreases, abnormal stress on the knee meniscus increases, causing meniscal degeneration through nitric oxide, as discussed above.

Calcification is common in meniscal degeneration ${ }^{32-34}$. Mechanical stress and inflammatory factors promote the overexpression of ANKH and ENPP1 in meniscal fibrochondrocytes, leading to increased intracellular calcium concentration or enhanced calcium signaling that affects the cell function, impairing the meniscal repair capacity ${ }^{35-37}$. Phosphocitrate can inhibit the proliferation of meniscal cells and calcification, delaying and reversing meniscal degeneration and osteoarthritis ${ }^{38}$. In this study, upregulated transition metal ion binding was observed, and calcium is a transition metal. Upregulated iron ion binding was also observed, and mice with hereditary hemochromatosis are more prone to knee osteoarthritis and meniscal degeneration due to iron overload $^{39}$.

In the pathway analysis, this study found that the upregulated pathways with the highest enrichment scores were type II diabetes mellitus, inflammatory mediator regulation of TRP channels, and AMPK signaling pathway. Insulin-like growth factors (IGFs) play an extremely important role in normal physiological activities, including musculoskeletal system development and meniscal repair ${ }^{40}$. Impaired insulin secretion leads to declination in the use of glucose by the cells, which in turn stimulates the AMPK signaling pathway, leading to enhanced catabolism and inhibited anabolism. The incidence of meniscal degeneration or osteoarthritis in patients with type II diabetes mellitus is higher than in normal individuals ${ }^{41}$. Damaged joints cause a series of reactions due to pro-inflammatory factors (such as IL-1 and TNF- $\alpha$ ) and activate the MAPK pathways, such as increased MMPs secretion, ADAMTS-4 enhanced activity, increased chemokine concentration, and increased nitric oxide synthesis, among others ${ }^{21,42,43}$.

Those results are supported by a study in humans that showed that meniscal degeneration involves the biological processes of immune response, inflammatory response, biomineral formation, and cell proliferation ${ }^{44}$. 


\begin{tabular}{|c|c|c|c|c|}
\hline GO.ID & Term & P value & Enrichment_Score & GENES \\
\hline GO:0000976 & $\begin{array}{l}\text { Transcription regulatory region sequence-specific DNA } \\
\text { binding }\end{array}$ & 0.00394022 & 2.404479912 & TFAP2D//MYOD1//HOXD13//GTF2B//NR5A1//KLF1 \\
\hline GO:1990837 & Sequence-specific double-stranded DNA binding & 0.00457772 & 2.339350843 & NR5A1//TFAP2D//MYOD1//HOXD13//GTF2B//KLF1 \\
\hline GO:0001618 & Virus receptor activity & 0.00458925 & 2.338258437 & SLC52A2//CLDN6 \\
\hline GO:0104005 & Hijacked molecular function & 0.00458925 & 2.338258437 & SLC52A2//CLDN6 \\
\hline GO:0046914 & Transition metal ion binding & 0.00552051 & 2.258020627 & $\begin{array}{l}\text { CYP2C33//CYP4A21//CYP17A1//ACP5//NR5A1//TRIM26// } \\
\text { TRIM55//TRIM40//S100A12//SEC23B }\end{array}$ \\
\hline GO:0005506 & Iron ion binding & 0.00764193 & 2.116796715 & ACP5//CYP2C33//CYP4A21//CYP17A1 \\
\hline GO:0003690 & Double-stranded DNA binding & 0.00832021 & 2.079865574 & NR5A1//TFAP2D//MYOD1//HOXD13//GTF2B//KLF1 \\
\hline GO:0001067 & Regulatory region nucleic acid binding & 0.01189735 & 1.92454989 & NR5A1//TFAP2D//MYOD1//HOXD13//GTF2B//KLF1 \\
\hline GO:0044212 & Transcription regulatory region DNA binding & 0.01189735 & 1.92454989 & NR5A1//TFAP2D//MYOD1//HOXD13//GTF2B//KLF1 \\
\hline GO:0000977 & $\begin{array}{l}\text { RNA polymerase II regulatory region sequence-specific DNA } \\
\text { binding }\end{array}$ & 0.01196138 & 1.922218857 & MYOD1//HOXD13//GTF2B//NR5A1//TFAP2D \\
\hline GO:0001012 & RNA polymerase II regulatory region DNA binding & 0.01196138 & 1.922218857 & TFAP2D//MYOD1//HOXD13//GTF2B//NR5A1 \\
\hline GO:0001158 & Enhancer sequence-specific DNA binding & 0.01223529 & 1.912385766 & NR5A1//HOXD13 \\
\hline GO:0004497 & Monooxygenase activity & 0.01493105 & 1.82590963 & CYP17A1//CYP4A21//CYP2C33 \\
\hline GO:0003705 & $\begin{array}{l}\text { Transcription factor activity, RNA polymerase II distal } \\
\text { enhancer sequence-specific binding }\end{array}$ & 0.01501133 & 1.823580805 & NR5A1//MYOD1 \\
\hline GO:0046872 & Metal ion binding & 0.01558956 & 1.80716619 & $\begin{array}{l}\text { LOC106505565//CYP2C33//CYP4A21//CYP17A1//CSN2// } \\
\text { TNNC2//KCNIP1//DLK2//S100A12//ACP5//NR5A1// } \\
\text { TRIM26//TRIM55//TRIM40//SEC23B//ACE2//GTF2B// } \\
\text { ADAM30 }\end{array}$ \\
\hline GO:0043169 & Cation binding & 0.01926006 & 1.715342436 & $\begin{array}{l}\text { LOC106505565//CYP2C33//CYP4A21//CYP17A1//CSN2// } \\
\text { TNNC2//KCNIP1//DLK2//S100A12//ACP5//NR5A1// } \\
\text { TRIM26//TRIM55//TRIM40//SEC23B//ACE2//GTF2B// } \\
\text { ADAM30 }\end{array}$ \\
\hline GO:0035326 & Enhancer binding & 0.0196297 & 1.707086296 & NR5A1//HOXD13 \\
\hline GO:0004866 & Endopeptidase inhibitor activity & 0.02517915 & 1.598959005 & ITIH2//COL7A1//CSN2 \\
\hline GO:0061135 & Endopeptidase regulator activity & 0.02925729 & 1.533765915 & ITIH2//COL7A1//CSN2 \\
\hline GO:0030414 & Peptidase inhibitor activity & 0.03032845 & 1.518149723 & ITIH2//COL7A1//CSN2 \\
\hline GO:0004857 & Enzyme inhibitor activity & 0.03165318 & 1.499582599 & SOCS2//ITIH2//COL7A1//CSN2 \\
\hline GO:0001047 & Core promoter binding & 0.03235762 & 1.49002349 & GTF2B//MYOD1 \\
\hline GO:0001653 & Peptide receptor activity & 0.03481855 & 1.458189356 & NPY2R//CCR10//HCRTR2 \\
\hline GO:0008528 & G-protein coupled peptide receptor activity & 0.03481855 & 1.458189356 & NPY2R//CCR10//HCRTR2 \\
\hline GO:0016705 & $\begin{array}{l}\text { Oxidoreductase activity, acting on paired donors, with incor- } \\
\text { poration or reduction of molecular oxygen }\end{array}$ & 0.03599219 & 1.443791785 & CYP17A1//CYP4A21//CYP2C33 \\
\hline GO:0020037 & Heme binding & 0.03599219 & 1.443791785 & CYP2C33//CYP4A21//CYP17A1 \\
\hline GO:0046906 & Tetrapyrrole binding & 0.03963483 & 1.401922989 & CYP2C33//CYP4A21//CYP17A1 \\
\hline GO:0061134 & Peptidase regulator activity & 0.04477304 & 1.348983405 & ITIH2//COL7A1//CSN2 \\
\hline GO:0004867 & Serine-type endopeptidase inhibitor activity & 0.04520724 & 1.344792035 & ITIH2//COL7A1 \\
\hline GO:0043167 & Ion binding & 0.04984315 & 1.302394546 & $\begin{array}{l}\text { LOC106505565//CYP2C33//CYP4A21//CYP17A1//CSN2// } \\
\text { TNNC2//KCNIP1//DLK2//S100A12//MYH7//NLRC3// } \\
\text { PTK2B//RAB33A//RAB25//NR5A1//ACP5//TRIM26// } \\
\text { TRIM55//TRIM40//SEC23B//FASN//ACE2//GTF2B// } \\
\text { ADAM30//DAO }\end{array}$ \\
\hline
\end{tabular}

Table 7. The molecular functions with up-regulated gene enrichment scores.

Despite the differences between humans and mini-pigs, the results of the involved pathways and genes are consistent between the two species.

There are some limitations to the present study. First, the experiment used the contralateral leg as a control group. The biomechanical characteristics of the contralateral leg also change during walking because of the affected leg, which in turn can cause some changes in gene expression. Therefore, it cannot be considered an ideal control group. Second, meniscal tissue is composed of a variety of cells; therefore, the DEGs are, in fact, the response of the overall tissue to modeling. The role of each cell constituent of the meniscus in degeneration is difficult to define clearly. Further research should be conducted through cell isolation culture or single-cell gene sequencing ${ }^{44,45}$. Third, the results obtained from animal experiments might differ from humans, suggesting only possible mechanisms for human meniscal degeneration.

\section{Conclusion}

Wuzhishan mini-pigs can be used as animal models for studying meniscus degeneration through the resection of the ACL and LCL. The present study provides some insight into the molecular mechanisms underlying meniscal degeneration. They might also reveal potential targets for meniscal degeneration treatment and early diagnosis. 
A

Sig GO terms of DE gene-MF

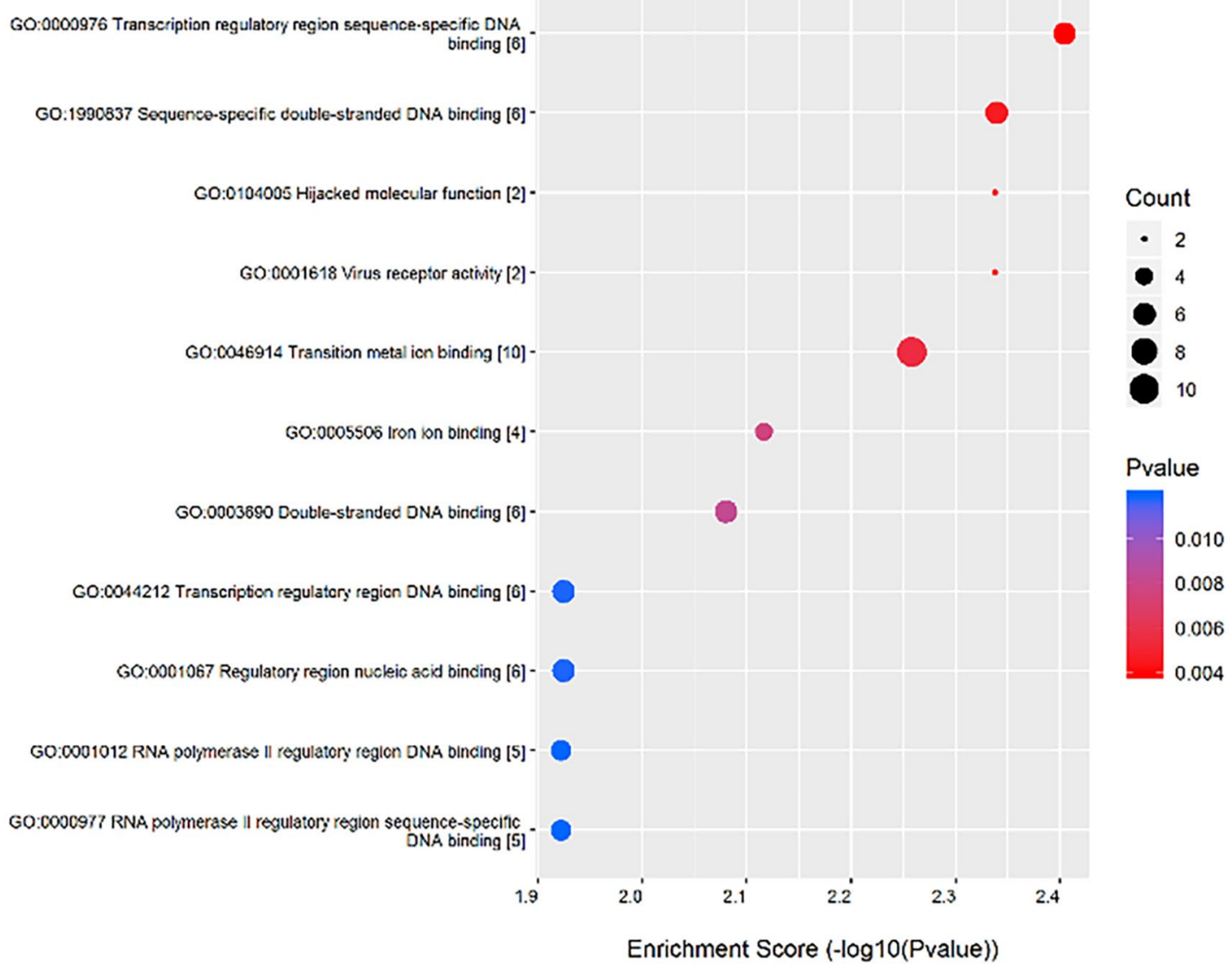

Sig GO terms of DE gene-MF

B

60:0003705 Transcription factor activity. RNA polymerase II distal enhancer sequence-specific binding [2]

GO:0005179 Hormone activity [3] -

GO:0035326 Enhancer binding [2]-

GO:0003700 DNA binding transcription factor activity [6] -

GO:0140110 Transcription regulator activity [6] -

GO:0042923 Neuropeptide binding [1]- .

$1.50^{\prime}$

$1.75^{\prime}$
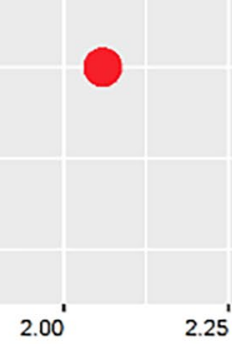

Count

- 1

- 2

- 3

4

5
6

Enrichment Score (- $\log 10$ (Pvalue))

Figure 5. The molecular function enrichment analyses for the differentially expressed genes (DEGs) analysed by Gene Ontology (GO). (A) The top ten molecular functions with upregulated Enrichment Score Dot Plot. (B) The molecular functions with upregulated Enrichment Score Dot Plot. 


\begin{tabular}{|l|l|l|l|l|}
\hline GO.ID & Term & P value & Enrichment_Score & Genes \\
\hline GO:0003705 & $\begin{array}{l}\text { Transcription factor activity, RNA polymerase II distal enhancer sequence- } \\
\text { specific binding }\end{array}$ & 0.00403475 & 2.394183037 & NR5A1//LEF1 \\
\hline GO:0005179 & Hormone activity & 0.00426023 & 2.370566551 & PDYN//TTR//MLN \\
\hline GO:0035326 & Enhancer binding & 0.00532467 & 2.273707091 & NR5A1//LEF1 \\
\hline GO:0003700 & DNA binding transcription factor activity & 0.00872967 & 2.05900229 & NR5A1//LEF1//DMRT1//ATF4//OTX2//ATF7 \\
\hline GO:0140110 & Transcription regulator activity & 0.02236277 & 1.650474363 & DMRT1//ATF4//OTX2//ATF7//NR5A1//LEF1 \\
\hline GO:0042923 & Neuropeptide binding & 0.04714984 & 1.326519782 & SSTR2 \\
\hline
\end{tabular}

Table 8. The molecular functions with up-regulated gene enrichment scores.

\begin{tabular}{|c|c|c|c|c|}
\hline Pathway ID & Definition & Fisher-P value & Enrichment_Score & Genes \\
\hline ssc04930 & Type II diabetes mellitus-Sus scrofa (pig) & 0.000759595 & 3.119418 & ABCC8//ADIPOQ//SLC2A4//SOCS2 \\
\hline ssc04742 & Taste transduction—Sus scrofa (pig) & 0.002858715 & 2.543829 & ADCY4//ADCY6//GABBR1//P2RY4 \\
\hline ssc04917 & Prolactin signaling pathway_-Sus scrofa (pig) & 0.003352503 & 2.474631 & CSN2//CYP17A1//PRL//SOCS2 \\
\hline ssc04211 & Longevity regulating pathway_-Sus scrofa (pig) & 0.007875137 & 2.103742 & ADCY4//ADCY6//ADIPOQ//ADIPOR2 \\
\hline ssc04913 & Ovarian steroidogenesis_-Sus scrofa (pig) & 0.009215694 & 2.035472 & ADCY4//ADCY6//CYP17A1 \\
\hline $\operatorname{ssc} 04080$ & Neuroactive ligand-receptor interaction—Sus scrofa (pig) & 0.01021098 & 1.990932 & $\begin{array}{l}\text { ADRA2C//GABBR1//HCRTR2//NPY2R//P2RY4//PRL// } \\
R X F P 1\end{array}$ \\
\hline ssc04750 & $\begin{array}{l}\text { Inflammatory mediator regulation of TRP channels_-Sus } \\
\text { scrofa (pig) }\end{array}$ & 0.01098087 & 1.959363 & ADCY4//ADCY6//CYP2C33//TRPA1 \\
\hline ssc00770 & Pantothenate and CoA biosynthesis_-Sus scrofa (pig) & 0.01205545 & 1.918817 & UPB1//VNN1 \\
\hline ssc04152 & AMPK signaling pathway—Sus scrofa (pig) & 0.02220957 & 1.65346 & ADIPOQ//ADIPOR2//FASN//SLC2A4 \\
\hline ssc04976 & Bile secretion-Sus scrofa (pig) & 0.02225764 & 1.652521 & $A B C B 11 / / A D C Y 4 / / A D C Y 6$ \\
\hline ssc04920 & Adipocytokine signaling pathway-Sus scrofa (pig) & 0.0249136 & 1.603563 & ADIPOQ//ADIPOR2//SLC2A4 \\
\hline ssc00983 & Drug metabolism—other enzymes-Sus scrofa (pig) & 0.03062382 & 1.513941 & CES1//UPB1 \\
\hline ssc04910 & Insulin signaling pathway—Sus scrofa (pig) & 0.0323184 & 1.49055 & FASN//PPP1R3D//SLC2A4//SOCS2 \\
\hline ssc05143 & African trypanosomiasis-Sus scrofa (pig) & 0.03438215 & 1.463667 & IDO2//LOC396781 \\
\hline ssc05414 & Dilated cardiomyopathy (DCM) - Sus scrofa (pig) & 0.0360517 & 1.443074 & ADCY4//ADCY6//LOC396781 \\
\hline ssc05340 & Primary immunodeficiency_Sus scrofa (pig) & 0.03632543 & 1.439789 & IL2RG//LOC396781 \\
\hline ssc04911 & Insulin secretion-Sus scrofa (pig) & 0.03717241 & 1.429779 & ABCC8//ADCY4//ADCY6 \\
\hline ssc04727 & GABAergic synapse-Sus scrofa (pig) & 0.03831094 & 1.416677 & $A D C Y 4 / / A D C Y 6 / / G A B B R 1$ \\
\hline ssc04072 & Phospholipase D signaling pathway_-Sus scrofa (pig) & 0.0386556 & 1.412788 & ADCY4//ADCY6//LOC396781//PTK2B \\
\hline ssc04974 & Protein digestion and absorption-Sus scrofa (pig) & 0.04064128 & 1.391033 & ACE2//COL7A1//SLC7A8 \\
\hline $\operatorname{ssc} 04912$ & GnRH signaling pathway-Sus scrofa (pig) & 0.04304219 & 1.366106 & $A D C Y 4 / / A D C Y 6 / / P T K 2 B$ \\
\hline ssc04914 & Progesterone-mediated oocyte maturation-Sus scrofa (pig) & 0.04304219 & 1.366106 & ADCY4//ADCY6//CPEB4 \\
\hline ssc05032 & Morphine addiction—Sus scrofa (pig) & 0.04551314 & 1.341863 & $A D C Y 4 / / A D C Y 6 / / G A B B R 1$ \\
\hline ssc01522 & Endocrine resistance-Sus scrofa (pig) & 0.0467747 & 1.329989 & ABCB11//ADCY4//ADCY6 \\
\hline
\end{tabular}

Table 9. The pathways with up-regulated gene enrichment scores.

\begin{tabular}{|l|l|l|l|l|}
\hline Pathway ID & Definition & Fisher-P value & Enrichment_Score & Genes \\
\hline ssc04918 & Thyroid hormone synthesis_Sus scrofa (pig) & 0.002669959 & 2.573495 & ATF4//TG//TTR \\
\hline ssc05030 & Cocaine addiction_Sus scrofa (pig) & 0.01468413 & 1.833152 & ATF4//PDYN \\
\hline ssc00980 & $\begin{array}{l}\text { Metabolism of xenobiotics by cytochrome P450_Sus scrofa } \\
\text { (pig) }\end{array}$ & 0.01528523 & 1.815728 & CYP2E1//EPHX1 \\
\hline ssc05134 & Legionellosis_Sus scrofa (pig) & 0.01844662 & 1.734083 & CD14//TLR5 \\
\hline ssc00561 & Glycerolipid metabolism_Sus scrofa (pig) & 0.02257206 & 1.646429 & AKR1B1//PNLIP \\
\hline ssc05204 & Chemical carcinogenesis_Sus scrofa (pig) & 0.02329364 & 1.632763 & CYP2E1//EPHX1 \\
\hline ssc05031 & Amphetamine addiction-Sus scrofa (pig) & 0.02627419 & 1.580471 & ATF4//PDYN \\
\hline ssc05221 & Acute myeloid leukemia-Sus scrofa (pig) & 0.02627419 & 1.580471 & CD14//LEF1 \\
\hline ssc04520 & Adherens junction-Sus scrofa (pig) & 0.03020581 & 1.519909 & LEF1//SSX2IP \\
\hline ssc05132 & Salmonella infection-Sus scrofa (pig) & 0.03961531 & 1.402137 & CD14//TLR5 \\
\hline ssc04974 & Protein digestion and absorption_Sus scrofa (pig) & 0.04516019 & 1.345244 & PGA5//SLC7A9 \\
\hline ssc04640 & Hematopoietic cell lineage-Sus scrofa (pig) & 0.04706952 & 1.32726 & CD14//GYPA \\
\hline
\end{tabular}

Table 10. The pathways with down-regulated gene enrichment scores. 
A

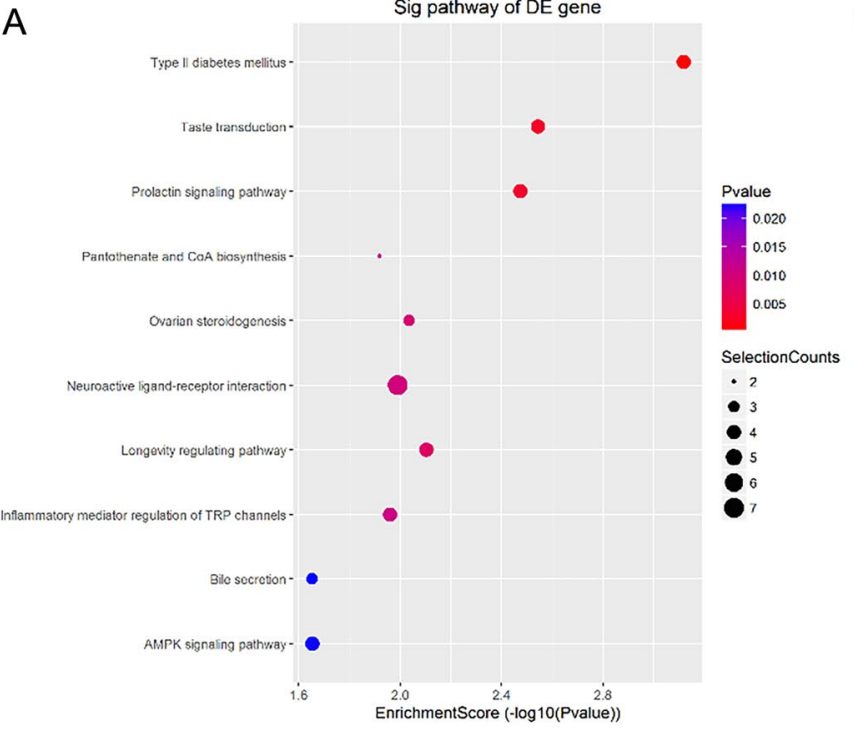

B

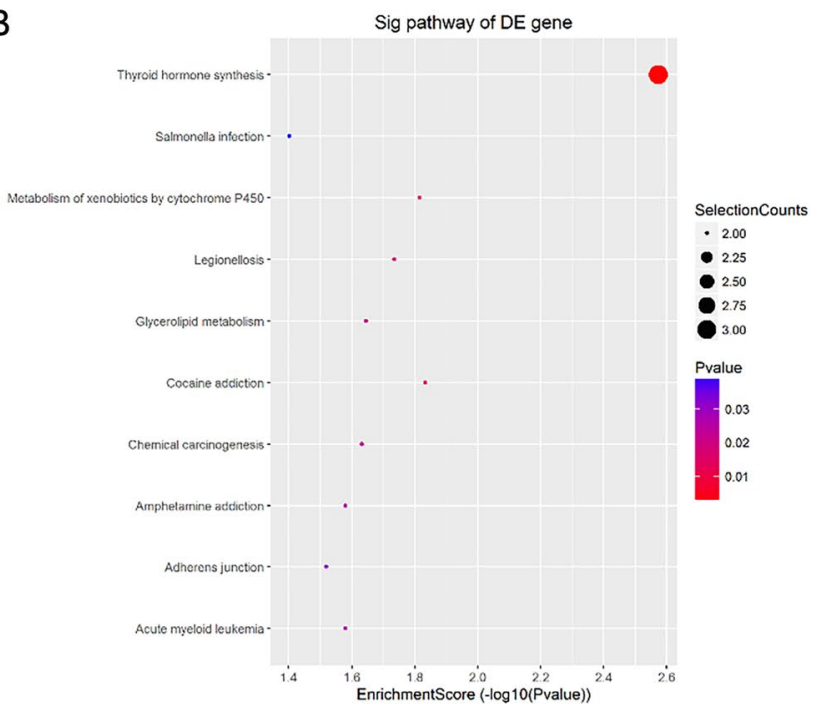

Figure 6. Pathways analyses for the differentially expressed genes (DEGs) analysed by Kyoto Encyclopedia of Genes and Genomes (KEGG). (A) The top ten pathways with upregulated Enrichment Score Dot Plot. (B) The top ten pathways with down-regulated Enrichment Score Dot Plot.

\begin{tabular}{|c|c|c|c|c|c|}
\hline Genbank accession & Gene symbol & Gene name & Description & Fold change by microarrays & Fold change by RT-PCR \\
\hline CD572197 & ABCC8 & $\begin{array}{l}\text { ATP binding cassette subfamily C } \\
\text { member } 8\end{array}$ & $\begin{array}{l}\text { ATP binding cassette subfam- } \\
\text { ily C member } 8 \text { [Source:HGNC } \\
\text { Symbol;Acc:HGNC:59] [ENS- } \\
\text { SSCT00000014612] }\end{array}$ & 2.332 & $3.370^{* *}$ \\
\hline XM_001926115 & TRPA1 & $\begin{array}{l}\text { Transient receptor potential cation } \\
\text { channel subfamily A member } 1\end{array}$ & $\begin{array}{l}\text { PREDICTED: Sus scrofa transient } \\
\text { receptor potential cation channel, sub- } \\
\text { family A, member 1 (TRPA1), mRNA } \\
\text { [XM_001926115] }\end{array}$ & 2.104 & $2.887^{\star *}$ \\
\hline NM_213926 & PRL & Prolactin & $\begin{array}{l}\text { Sus scrofa prolactin (PRL), mRNA } \\
\text { [NM_213926] }\end{array}$ & 2.040 & $2.775^{* *}$ \\
\hline JX092267 & ADIPOQ & $\begin{array}{l}\text { Adiponectin, C1Q and collagen } \\
\text { domain containing }\end{array}$ & $\begin{array}{l}\text { Rep: Adiponectin_Sus scrofa (Pig), } \\
\text { complete [TC535135] }\end{array}$ & 2.667 & $2.955^{* *}$ \\
\hline AB005285 & SLC2A4 & Solute carrier family 2 member 4 & GB & 2.520 & $3.091^{\star *}$ \\
\hline NM_214428 & CYP17A1 & Cytochrome P450 17A1 & $\begin{array}{l}\text { Sus scrofa cytochrome P450 17A1 } \\
\text { (CYP17A1), mRNA [NM_214428] }\end{array}$ & 2.059 & $2.962^{* *}$ \\
\hline XM_013978180 & ADCY4 & Adenylate cyclase 4 & $\begin{array}{l}\text { adenylate cyclase } 4 \text { [Source:HGNC } \\
\text { Symbol;Acc:HGNC:235] [ENS- } \\
\text { SSCT00000002224] }\end{array}$ & 2.443 & $2.886^{* *}$ \\
\hline NM_214209 & ACP5 & Acid phosphatase 5 , tartrate resistant & $\begin{array}{l}\text { Sus scrofa acid phosphatase 5, } \\
\text { tartrate resistant (ACP5), mRNA } \\
\text { [NM_214209] }\end{array}$ & 2.459 & $2.816^{* *}$ \\
\hline NM_214111 & DMRT1 & $\begin{array}{l}\text { Doublesex and mab-3 related tran- } \\
\text { scription factor } 1\end{array}$ & $\begin{array}{l}\text { Sus scrofa doublesex and mab-3 } \\
\text { related transcription factor 1 } \\
\text { (DMRT1), mRNA [NM_214111] }\end{array}$ & -2.050 & -1.356 \\
\hline NM_001167795 & ACTA1 & Actin, alpha 1 , skeletal muscle & $\begin{array}{l}\text { Sus scrofa actin, alpha 1, skel- } \\
\text { etal muscle (ACTA1), mRNA } \\
\text { [NM_001167795] }\end{array}$ & -2.139 & -1.337 \\
\hline NM_001011694 & SSTR2 & Somatostatin receptor 2 & $\begin{array}{l}\text { Sus scrofa somatostatin receptor } 2 \\
\text { (SSTR2), mRNA [NM_001011694] }\end{array}$ & -2.130 & -1.420 \\
\hline NM_214355 & EPHX1 & Epoxide hydrolase 1 & $\begin{array}{l}\text { Sus scrofa epoxide hydrolase 1 } \\
\text { (EPHX1), mRNA [NM_214355] }\end{array}$ & -2.082 & $-1.488^{*}$ \\
\hline
\end{tabular}

Table 11. The expression profiles of selected genes from microarray and RT-PCR. Positive number indicates elevated expression (fold) in the meniscus of the experimental group (Ba group) compared to the meniscus of the control group (Aa group). Negative number indicates decreased expression (fold) in the meniscus of the experimental group (Ba group) compared to the meniscus of the control group (Aa group). ${ }^{*}<<0.05,{ }^{* *} \mathrm{p}<0.01$ (versus sham-operated controls). 


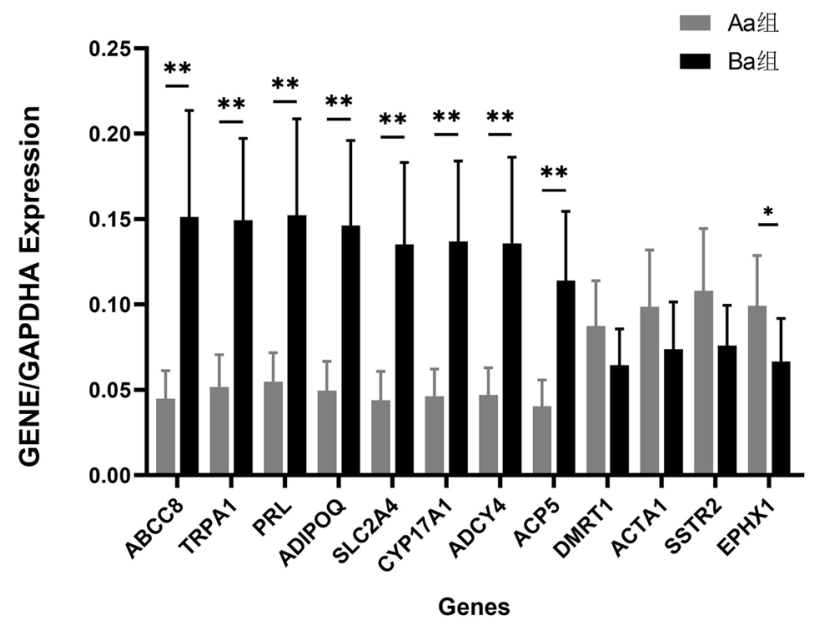

Figure 7. The selected 12 DEGs were validated by RT-PCR. Error bars indicated mean \pm standard errors of the mean. $\left({ }^{*} P<0.05\right)$.

\section{Data availability}

The datasets used and/or analyzed during the current study are available from the corresponding author on reasonable request.

Received: 26 May 2020; Accepted: 20 November 2020

Published online: 08 December 2020

\section{References}

1. Yeow, C. H., Lau, S. T., Lee, P. V. \& Goh, J. C. Damage and degenerative changes in menisci-covered and exposed tibial osteochondral regions after simulated landing impact compression-a porcine study. J. Orthop. Res. 27, 1100-1108 (2009).

2. Iijima, H. et al. Immature articular cartilage and subchondral bone covered by menisci are potentially susceptive to mechanical load. BMC Musculoskelet. Disord. 15, 101 (2014).

3. Iijima, H. et al. Destabilization of the medial meniscus leads to subchondral bone defects and site-specific cartilage degeneration in an experimental rat model. Osteoarthr. Cartil. 22, 1036-1043 (2014).

4. Fischenich, K. M., Lewis, J., Kindsfater, K. A., Bailey, T. S. \& Haut Donahue, T. L. Effects of degeneration on the compressive and tensile properties of human meniscus. J. Biomech. 48, 1407-1411 (2015).

5. Bendele, A. M. Animal models of osteoarthritis. J. Musculoskelet. Neuronal. Interact. 1, 363-376 (2001).

6. Sun, Y. et al. Expression of phosphocitrate-targeted genes in osteoarthritis menisci. Biomed. Res. Int. 2014, 210469 (2014).

7. Kapadia, R. D. et al. Meniscal ossification in spontaneous osteoarthritis in the guinea-pig. Osteoarthr. Cartil. 8, 374-377 (2000).

8. Cheung, H. S., Sallis, J. D., Demadis, K. D. \& Wierzbicki, A. Phosphocitrate blocks calcification-induced articular joint degeneration in a guinea pig model. Arthritis Rheum 54, 2452-2461 (2006).

9. Sun, Y. et al. Phosphocitrate is potentially a disease-modifying drug for noncrystal-associated osteoarthritis. Biomed. Res. Int. 2013, 326267 (2013).

10. Libicher, M., Ivancic, M., Hoffmann, M. \& Wenz, W. Early changes in experimental osteoarthritis using the Pond-Nuki dog model: technical procedure and initial results of in vivo MR imaging. Eur. Radiol. 15, 390-394 (2005).

11. Kreinest, M. et al. A porcine animal model for early meniscal degeneration: analysis of histology, gene expression and magnetic resonance imaging six months after resection of the anterior cruciate ligament. PLoS ONE 11, e0159331 (2016).

12. Zhang, R. et al. Gene expression analyses of subchondral bone in early experimental osteoarthritis by microarray. PLoS ONE 7 , e32356 (2012).

13. Kanehisa, M. \& Goto, S. KEGG: kyoto encyclopedia of genes and genomes. Nucleic Acids Res. 28, 27-30 (2000).

14. Kanehisa, M., Sato, Y., Furumichi, M., Morishima, K. \& Tanabe, M. New approach for understanding genome variations in KEGG. Nucleic Acids Res. 47, D590-d595 (2019).

15. Kanehisa, M. Toward understanding the origin and evolution of cellular organisms. Protein Sci. Publ. Protein Soc. 28, 1947-1951 (2019).

16. Papageorgiou, C. D. et al. The biomechanical interdependence between the anterior cruciate ligament replacement graft and the medial meniscus. Am. J. Sports Med. 29, 226-231 (2001).

17. Cerejo, R. et al. The influence of alignment on risk of knee osteoarthritis progression according to baseline stage of disease. Arthritis Rheum. 46, 2632-2636 (2002).

18. Reisig, G. et al. Osteoarthritis in the knee joints of gottingen minipigs after resection of the anterior cruciate ligament? Missing correlation of MRI, gene and protein expression with histological scoring. PLoS ONE 11, e0165897 (2016).

19. Rai, M. F., Tycksen, E. D., Sandell, L. J. \& Brophy, R. H. Advantages of RNA-seq compared to RNA microarrays for transcriptome profiling of anterior cruciate ligament tears. J. Orthop. Res. 36, 484-497 (2018).

20. Killian, M. L., Zielinska, B., Gupta, T. \& Haut Donahue, T. L. In vitro inhibition of compression-induced catabolic gene expression in meniscal explants following treatment with IL-1 receptor antagonist. J. Orthop. Sci. 16, 212-220 (2011).

21. Cook, A. E., Cook, J. L. \& Stoker, A. M. Metabolic responses of meniscus to IL-1beta. J. Knee Surg. 31, 834-840 (2018).

22. Shen, C., Yan, J., Erkocak, O. F., Zheng, X. F. \& Chen, X. D. Nitric oxide inhibits autophagy via suppression of JNK in meniscal cells. Rheumatology (Oxford) 53, 1022-1033 (2014).

23. Takahashi, K. et al. Hyaluronan suppressed nitric oxide production in the meniscus and synovium of rabbit osteoarthritis model. J. Orthop. Res. 19, 500-503 (2001).

24. Hafelein, K., Preusse-Prange, A., Behrendt, P. \& Kurz, B. Selenium reduces early signs of tumor necrosis factor alpha-induced meniscal tissue degradation. Biol. Trace Elem. Res. 177, 80-89 (2017). 
25. Behrendt, P. et al. IL-10 ameliorates TNF-alpha induced meniscus degeneration in mature meniscal tissue in vitro. BMC Musculoskelet. Disord. 18, 197 (2017).

26. Choi, C. J., Choi, Y. J., Song, I. B. \& Choi, C. H. Characteristics of radial tears in the posterior horn of the medial meniscus compared to horizontal tears. Clin. Orthop. Surg. 3, 128-132 (2011).

27. Hwang, B. Y. et al. Risk factors for medial meniscus posterior root tear. Am. J. Sports Med. 40, 1606-1610 (2012).

28. Zhang, F. et al. Risk factors for meniscal body extrusion on MRI in subjects free of radiographic knee osteoarthritis: longitudinal data from the Osteoarthritis Initiative. Osteoarthr. Cartil. 24, 801-806 (2016).

29. Lange, A. K. et al. Degenerative meniscus tears and mobility impairment in women with knee osteoarthritis. Osteoarthr. Cartil. 15, 701-708 (2007).

30. Sliwinski, L. et al. Effects of fenoterol on the skeletal system depend on the androgen level. Pharmacol. Rep. 69, 260-267 (2017).

31. Mobley, C. B. et al. Effects of testosterone treatment on markers of skeletal muscle ribosome biogenesis. Andrologia 48, 967-977 (2016).

32. Pauli, C. et al. Macroscopic and histopathologic analysis of human knee menisci in aging and osteoarthritis. Osteoarthr. Cartil. 19, 1132-1141 (2011).

33. Sun, Y. \& Mauerhan, D. R. Meniscal calcification, pathogenesis and implications. Curr. Opin. Rheumatol. 24, 152-157 (2012).

34. Stone, A. V. et al. Osteoarthritic changes in vervet monkey knees correlate with meniscus degradation and increased matrix metalloproteinase and cytokine secretion. Osteoarthr. Cartil. 23, 1780-1789 (2015).

35. Han, W. M. et al. Impact of cellular microenvironment and mechanical perturbation on calcium signalling in meniscus fibrochondrocytes. Eur. Cell Mater. 27, 321-331 (2014).

36. McNulty, A. L., Rothfusz, N. E., Leddy, H. A. \& Guilak, F. Synovial fluid concentrations and relative potency of interleukin-1 alpha and beta in cartilage and meniscus degradation. J. Orthop. Res. 31, 1039-1045 (2013).

37. Du, G. et al. Abnormal mechanical loading induces cartilage degeneration by accelerating meniscus hypertrophy and mineralization after ACL injuries in vivo. Am. J. Sports Med. 44, 652-663 (2016).

38. Sun, Y. et al. Biological activities of phosphocitrate: a potential meniscal protective agent. Biomed. Res. Int. 2013, 726581 (2013).

39. Camacho, A. et al. Iron overload in a murine model of hereditary hemochromatosis is associated with accelerated progression of osteoarthritis under mechanical stress. Osteoarthr. Cartil. 24, 494-502 (2016).

40. Katsuragawa, Y. et al. Changes of human menisci in osteoarthritic knee joints. Osteoarthr. Cartil. 18, 1133-1143 (2010).

41. Neumann, J. et al. Diabetics show accelerated progression of knee cartilage and meniscal lesions: data from the osteoarthritis initiative. Skelet. Radiol. 48, 919-930 (2019).

42. Hennerbichler, A., Moutos, F. T., Hennerbichler, D., Weinberg, J. B. \& Guilak, F. Interleukin-1 and tumor necrosis factor alpha inhibit repair of the porcine meniscus in vitro. Osteoarthr. Cartil. 15, 1053-1060 (2007).

43. Stone, A. V. et al. Pro-inflammatory stimulation of meniscus cells increases production of matrix metalloproteinases and additional catabolic factors involved in osteoarthritis pathogenesis. Osteoarthr. Cartil. 22, 264-274 (2014).

44. Sun, Y. et al. Analysis of meniscal degeneration and meniscal gene expression. BMC Musculoskelet. Disord. 11, 19 (2010).

45. Aoki, T. et al. Single-cell transcriptome analysis reveals disease-defining T-cell subsets in the tumor microenvironment of classic Hodgkin lymphoma. Cancer Discov. 10, 406-421 (2020).

\section{Acknowledgements}

We thank KangChen Bio-tech, Shanghai, China, for performing the microarray experiments and for their assistance with microarray data analysis; we also thank the Institute of Animal Science and Veterinary Medicine, Hainan Academy of Agricultural Sciences, Haikou, China, for providing useful technical assistance. In addition, we thank Xiaolong Xiong, Jindian Tan, Qiao Lin, and Hui Li for their assistance with the animal experiments.

\section{Author contributions}

Y.L. and J.L.: conceptualization, supervision, project administration. Y.F.: conceptualization, methodology, formal analysis, writing_original draft, writing — review and editing. H.H.: methodology, writing—review and editing. G.Z. and Q.W.: methodology. F.G. and C.L.: formal analysis. All authors reviewed the manuscript.

\section{Funding}

This work was supported by the Key R\&D plan of Hainan Province, China [Item No. ZDYF2017112].

\section{Competing interests}

The authors declare no competing interests.

\section{Additional information}

Correspondence and requests for materials should be addressed to Y.L. or J.L.

Reprints and permissions information is available at www.nature.com/reprints.

Publisher's note Springer Nature remains neutral with regard to jurisdictional claims in published maps and institutional affiliations.

Open Access This article is licensed under a Creative Commons Attribution 4.0 International License, which permits use, sharing, adaptation, distribution and reproduction in any medium or format, as long as you give appropriate credit to the original author(s) and the source, provide a link to the Creative Commons licence, and indicate if changes were made. The images or other third party material in this article are included in the article's Creative Commons licence, unless indicated otherwise in a credit line to the material. If material is not included in the article's Creative Commons licence and your intended use is not permitted by statutory regulation or exceeds the permitted use, you will need to obtain permission directly from the copyright holder. To view a copy of this licence, visit http://creativecommons.org/licenses/by/4.0/.

(C) The Author(s) 2020 